\title{
ARIADNE: Agnostic Reconfiguration In A Disconnected Network Environment
}

\author{
Konstantinos Aisopos ${ }^{\dagger \S}$, Andrew DeOrio ${ }^{\ddagger}$, Li-Shiuan $\mathrm{Peh}^{\S}$, and Valeria Bertacco ${ }^{\ddagger}$ \\ ${ }^{\dagger}$ Princeton University ${ }^{\S}$ Massachusetts Institute of Technology ${ }^{\ddagger}$ University of Michigan
}

\begin{abstract}
Extreme transistor technology scaling is causing increasing concerns in device reliability: the expected lifetime of individual transistors in complex chips is quickly decreasing, and the problem is expected to worsen at future technology nodes. With complex designs increasingly relying on Networks-on-Chip (NoCs) for on-chip data transfers, a NoC must continue to operate even in the face of many transistor failures. Specifically, it must be able to reconfigure and reroute packets around faults to enable continued operation, i.e., generate new routing paths to replace the old ones upon a failure. In addition to these reliability requirements, NoCs must maintain low latency and high throughput at very low area budget.

In this work, we propose a distributed reconfiguration solution named Ariadne, targeting large, aggressively scaled, unreliable NoCs. Ariadne utilizes up*/down* for fast routing at high bandwidth, and upon any number of concurrent network failures in any location, it reconfigures to discover new resilient paths to connect the surviving nodes. Experimental results show that Ariadne provides a $40 \%-140 \%$ latency improvement (when subject to 50 faults in a 64-node NoC) over other on-chip state-of-the-art fault tolerant solutions, while meeting the low area budget of on-chip routers with an overhead of just $1.97 \%$.
\end{abstract}

Keywords-NoC; resilience; reconfiguration; distributed

\section{INTRODUCTION}

Aggressive transistor scaling continues to increase integration capacity with each new technology node. With more transistors comes the need for modular communication architectures. Networks-on-Chip (NoCs), which offer distributed communication via a set of connected routers, are becoming more popular, as indicated by some recent designs, such as the Tile64 [4] and TERAFlops [30]. The NoCs of such manycore chips have to meet tough latency and throughput targets, in the face of stringent area and power budgets. Unfortunately, as critical dimensions shrink, reliability degrades as well. This highly scaled, unstable silicon demands new solutions that can gracefully handle permanent failures, occurring due to transistor wear-out, at any time during the life of the chip [8]. As the sole medium of communication, it is critical that a failure in the network does not cause an entire chip to fail.

Most current approaches for NoC reliability are only effective in overcoming a limited number of failures and specific fault patterns. Yet, a large number of transistors can fail in a many-core chip at advanced technology nodes $[7,8]$, resulting in many faults in the NoC. In addition, the locations of these faults are unpredictable and irregular in nature [11] and can thus lead to deadlock-prone irregular network topologies. Thus, any viable solution for failure-prone networks requires that the surviving nodes coordinate to reconfigure and replace the routing algorithm with a new one upon each new failure, by discovering the underlying topology and establishing deadlock-free routes.

Contributions. A fault-tolerant interconnect should address three critical metrics: reliability, performance, and area. In this work we propose Ariadne ${ }^{1}$, a novel network reconfiguration algorithm, providing unlimited system robustness and high performance, within a very low area budget. Ariadne is agnostic to the underlying topology: it can operate on any irregular topology resulting from an initial regular topology where any number of links have failed. Ariadne discovers paths among all connected nodes, and then utilizes up*/down* to provide highly adaptive and deadlock-free routing. It achieves this through a novel distributed algorithm implemented in hardware. This algorithm is designed to minimize communication among nodes, thus lowering its silicon area footprint. Ariadne addresses the above critical metrics as follows:

- Reliability: Ariadne guarantees connectivity among all surviving nodes in the network, in the face of unlimited faults at any location. That is, if a path between two nodes exists, the algorithm will enable at least one deadlock-free route between them.

- Performance: During normal operation (after recovering from faults), Ariadne achieves low latency and high throughput routing without Virtual Channels (VCs). This implies that no VC needs to be reserved for deadlock avoidance; all VCs can be used by all available routing paths, resulting in a 40\%-140\% latency improvement over state-of-the-art fault tolerant solutions [14, 25] (latency improvement measured at 50 faults in a 64-node $\mathrm{NoC}$ ).

- Area: Our distributed design requires only a small amount of hardware modifications and additional wiring to realize reconfiguration. This results in a low $1.97 \%$ area overhead over a baseline, non-reliable NoC.

This paper is organized as follows: Section II motivates the need for resilient NoCs that can handle many wear-out faults, and Section III presents the related work. Section IV details Ariadne, while Section V discusses the architectural modifications required to implement it. Then, Section VI offers our experimental results. Section VII discusses how deadlockfree execution is guaranteed in the face of runtime faults, as well as off-chip implementations of up*/down*. Finally, Section VIII concludes the paper.

\footnotetext{
${ }^{1}$ The name originates from Princess Ariadne from Greek mythology, who gave Theseus a ball of thread to help him find his way in the Minotaur's labyrinth. Similarly, our algorithm helps packets find their way in the labyrinth-like topology of a faulty network.
} 


\section{Motivation}

Recent studies project that there will be many transistor failures during the lifetime of many-core chips fabricated at advanced technology nodes. Researchers have characterized the impact of technology scaling on device reliability in processors [28] and Networks-on-Chips (NoCs) [3], and indicate that the number of permanent failures is expected to increase. Borkar of Intel expects that at future technology nodes $20 \%$ of transistors in chip multiprocessors will be unusable due to variations of the manufacturing process, while an additional $10 \%$ of transistors will eventually fail over the lifetime of the chip due to wear-out $[7,8]$.

To demonstrate the architectural impact of such high number of transistor failures in the $\mathrm{NoC}$, we developed an architecturelevel fault model, similar to the fault model used in Vicis [15], that maps gate-level injected faults to link-level faults. Initially, we synthesize and place-and-route a router design similar to that of [15], consisting of 20,413 gates. Then, we inject faults to its netlist using a random distribution, weighted by the area of each gate, in order to model the increased vulnerability of complex gates comprising more transistors, consistently with the breakdown patterns found experimentally by Keane, et al. [19]. For statistical confidence, we inject a total of 1,000 different fault configurations over a wide range of simultaneous gate faults. Then, we test each faulty netlist obtained to determine which links remained functional. This way, our fault model does not only model wiring failures, but also any failure within the router that results in nonfunctional communication links. For instance, if a gate failure disables a flit buffer of an output port, the corresponding output link will also be marked as faulty. Figure 1 shows the average number of link faults as a function of gate faults (error bars showing min and $\max$ ) when applying our fault model to an $8 \times 8$ mesh of routers. Note that even for just 30 gate faults, 5 to 50 network links are expected to

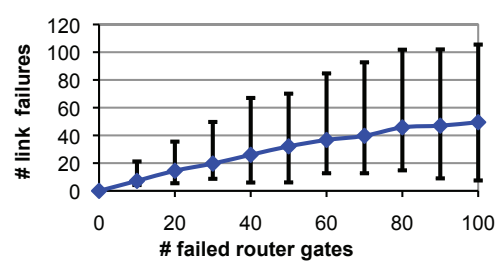

Fig. 1: Link vs. gate faults. fail, resulting in a highly impaired network. These wear-out link failures have no predictable pattern and can occur anywhere on-chip [11], thus leading to highly irregular network topologies.

In short, there is a strong need for low-overhead reliable solutions for NoCs that are capable to deliver low latency and high throughput in the presence of many irregular link failures and potentially complete node failures, since routers will become disconnected once all adjacent links have failed. However, we note that the duration of the reconfiguration process does not need to be optimal. A reconfiguration latency even in the order of milliseconds will not affect overall performance, since it only occurs upon a permanent link failure.

\section{RELATED WORK}

There has been substantial research on resilient NoCs [11, 15]. Here, we focus on prior works that tackle routing in the face of link failures. First, we classify them based on the type of fault targeted and whether a bounded or unbounded number of faults can be sustained. Then, we focus on solutions that can tolerate unbounded faults without pattern constraints and discuss current off-chip and on-chip approaches, indicating how they differ in their design constraints. Our focus in this paper is to achieve resilient routing on-chip in the face of many permanent faults, by providing a solution that reconfigures the network and updates the routing algorithm upon each failure.

Bounded number of faults. Early work on reliable routing targeted few link failures, such as 1-fault tolerance in Dally's reliable router [12], (n-1) fault tolerant $n$-dimensional meshes [16]. Works as in $[17,18,29]$ leverage additional virtual channels to work around a few link failures. There has also been prior art that tackles the problem by flooding packets to random neighbors, hoping that they will eventually reach their destination $[6,24]$. Such approaches result in very low throughput during normal operation.

Unbounded number of faults with pattern constraints. Other approaches place requirements on the configuration of faults, requiring them to be contained in convex regions [9, 31], L or T shape regions [9], or faulty polygons [20]. Oftentimes the faulty region must be expanded to include non-faulty routers; as a result, non-faulty routers and links are disabled only to satisfy the algorithm's constraints.

Unbounded number of faults, no pattern constraints. Table I qualitatively compares resilient routing algorithms that can tolerate unbounded faults without pattern constraints, based on critical evaluation metrics, which heavily depend on the reconfiguration algorithm. Reconfiguration is the process of generating a new routing algorithm (whenever a new link fault is detected) to replace the current one. Given a faulty topology, the reliability and performance of a routing algorithm can be optimized with a sophisticated reconfiguration algorithm, but complexity translates to high area overhead. Off-chip reconfiguration algorithms run in software and can perform powerful optimizations, but communicating the global view of the surviving topology to a central node to run the reconfiguration software requires expensive dedicated hardware. By contrast, on-chip solutions must be designed to meet tight on-chip area budgets, thus achieving high performance or high reliability is a challenge.

a) Off-chip solutions. Off chip networks, such as clusters and Networks-Of-Workstations, first tackled the reliability challenge of unconstrained faults. A number of resilient routing algorithms that can be applied to any irregular topology (i.e., a topology that survived after a number of random faults in network links) has been proposed in this domain, including up*/down* (introduced in Autonet) [27], segmentbased routing [23], FX routing [26], L-turn [21], and smartrouting [10]. During reconfiguration, the surviving topology is communicated to a central node, which runs the recon- 


\begin{tabular}{|c|c|c|c|c|c|}
\hline \multirow{2}{*}{ bounded faults } & \multirow{2}{*}{\multicolumn{2}{|c|}{ early work $[12,16]$, VCs $[17,18,29]$ flooding $[6,24]$}} & reliability & performance & area \\
\hline & & & limited & $\mathrm{n} / \mathrm{a}$ & $\mathrm{n} / \mathrm{a}$ \\
\hline \multirow{5}{*}{$\begin{array}{c}\text { pattern constraints } \\
\text { unbounded faults } \\
\text { without } \\
\text { pattern constraints }\end{array}$} & \multicolumn{2}{|c|}{ convex $[9,31], \mathrm{L}$ or $\mathrm{T}[9]$, polygons $[20]$} & limited & $\mathrm{n} / \mathrm{a}$ & $\mathrm{n} / \mathrm{a}$ \\
\hline & \multicolumn{2}{|c|}{ off-chip routing $[10,21,23,26,27]$} & high & good & excessive \\
\hline & \multirow{3}{*}{$\begin{array}{l}\text { on-chip } \\
\text { routing }\end{array}$} & Immunet [25] & high & bad & high \\
\hline & & Vicis [14] & limited & bad & low \\
\hline & & Ariadne (proposed) & high & good & low \\
\hline
\end{tabular}

TABLE I: Resilient routing landscape. Qualitative comparison of resilient solutions.

figuration algorithm in software. Using global knowledge of the functional links, the software computes new routing tables and communicates them back to each node. As we discuss in Section VII-C, centralized approaches lead to excessive area overhead for on-chip routers (estimated at 23.2\%).

b) On-chip solutions. On chip networks have a tight area and power budget, necessitating simple router structures. Reconfiguration is implemented completely in hardware and thus must be achievable with a simple Finite State Machine (FSM). There are two recent on-chip proposals that tackle the problem of unconstrained faults: Immunet [25] and Vicis [15]:

Immunet [25] routes packets fully adaptively towards their destinations, based on buffer availability. If necessary, packets switch to a reserved escape VC that guarantees that they will reach their destination and avoid faulty links. This VC is aware of the fault locations and routes deterministically in a ring through every node. Upon reconfiguration, a new ring that connects all surviving nodes is formed with a single broadcast, and all in-transit packets drain out via this ring, before updating the routing tables. While the ring guarantees delivery, it dramatically increases latency, since it must remain active during normal operation to ensure deadlock freedom. In addition, the design requires three routing tables per router, resulting in high area (storage) overhead.

Vicis [15] proposes a low overhead routing algorithm [14] to cope with an unbounded number of faults, by using a heuristic solution that makes exceptions to the odd-even turn model to maximize connectivity in meshes and tori. It utilizes the turn model during fault-free operation, but upon the occurrence of a fault, reconfiguration re-enables turns that were previously disabled by the routing algorithm to re-connect nodes that have been disconnected by the fault. As we show in Section VI, these exceptions sometimes result in deadlocked routing paths, especially in situations with large numbers of faults. Moreover, its deterministic nature does not exploit all possible routes, thus limiting performance during normal operation.

In this work, we propose the Ariadne reconfiguration algorithm to realize up*/down* on-chip in a fully-distributed manner. Up*/down* offers the unlimited robustness of Immunet, and higher performance (low latency and high throughput) than both Immunet and Vicis during normal operation, since no virtual channels are restricted to deterministic routing. Using a synchronization mechanism that leverages the global clock to guarantee atomicity and minimize communication among nodes, Ariadne reconfigures up*/down* (upon a fault) in an area budget three times lower than Immunet and comparable to Vicis (Section VI-D). Its implementation requires only a few gates and a single wire per port.

\section{ARIADNE ALgORITHM}

Permanent transistor faults may cause link failures that modify the topology of a NoC. Though the initial topology of a NoC is usually regular, after a number of link failures, nodes will be connected through a random irregular topology. Ariadne is a reconfiguration algorithm that is invoked upon a permanent link failure (e.g., due to transistor wear-out), and it is agnostic to the topology, since it includes a discovery phase of the underlying network to update the routing tables with new deadlock-free paths. In a network of $\mathrm{N}$ nodes, the reconfiguration procedure consists of $\mathrm{N}$ broadcasts, taking up to $\mathrm{N}^{2}$ cycles. The procedure may run in a partially or fully connected network, and guarantees that after $\mathrm{N}^{2}$ cycles every node will know the output port(s) to route to any connected destination ${ }^{2}$. Thereafter, network operation resumes normally.

Ariadne leverages up*/down* routing ${ }^{3}$, a deadlock-free algorithm that can operate on any irregular topology [27]. Up*/down* requires each link to be assigned a direction: up or down. It then disallows those paths that include traversing a down link followed by an up link. This way, all cyclic dependencies are broken. In Section IV-A, we describe our distributed reconfiguration algorithm that assigns a direction to each link, thus allowing up*/down* routing to be applied after reconfiguration. The algorithm then explores the new topology and fills the routing tables with resilient paths connecting all surviving nodes. A key cornerstone of our reconfiguration algorithm is that it is fully distributed, relying on a single atomic broadcast by each node to assign all link directions and to explore the underlying topology, as we describe in Section IV-B. This simple broadcast scheme makes for a very lightweight hardware implementation (detailed in Section V).

\section{A. Reconfiguration Algorithm}

The reconfiguration algorithm works as follows: each node, in turn, broadcasts a 1-bit reconfiguration flag to all nodes. The first node to broadcast is the node that detected the fault in the network, and it becomes the initiator (root node) of the reconfiguration process. Upon receiving the reconfiguration flag broadcasted from the root node, each node performs the following actions (Figure 2):

\footnotetext{
${ }^{2}$ Reconfiguration delay is not a concern, since the performance overhead induced by reconfiguration can only occur as many times as the link count, throughout the lifetime of the chip.

${ }^{3} \mathrm{Up} * /$ down* is a routing algorithm designed for irregular networks, thus not optimal for regular networks (i.e., a mesh). A potential optimization is to leverage DOR routing while the NoC is fault-free (regular), and switch to up*/down* once the first fault occurs. Note that transitioning from a routing algorithm to another may introduce deadlocks, which can be prevented as discussed in Section VII-B.
} 


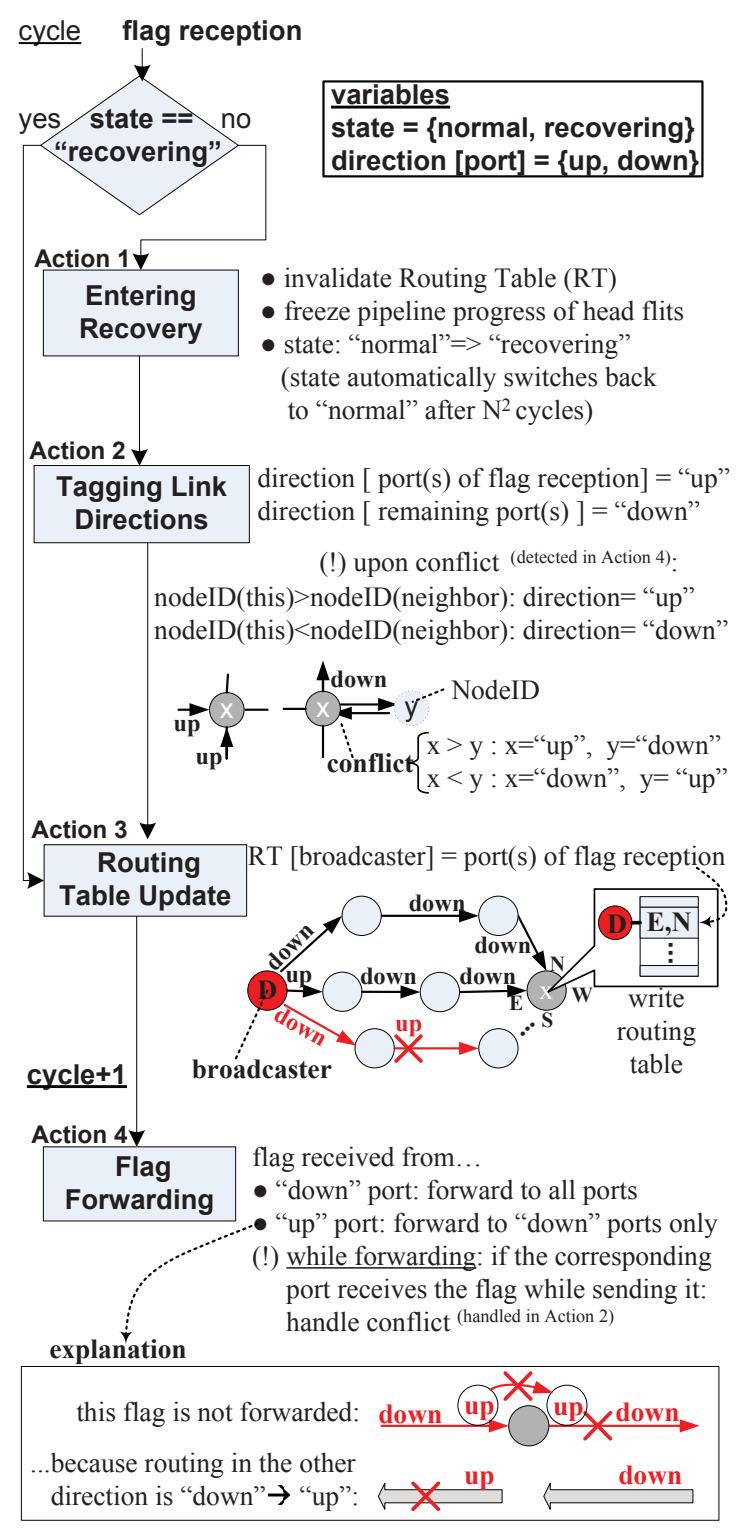

Fig. 2: Ariadne reconfiguration algorithm: Actions performed upon reception of the reconfiguration flag.

- Action1. Entering Recovery: enters recovery mode, invalidates the old routing paths, and freezes head flits.

- Action2. Tagging Link Directions: marks all links adjacent to the node either as up or down.

- Action3. Routing Table Update: informs routing table of which port(s) can be used to reach the node that initiated the broadcast.

- Action4. Flag Forwarding: forwards the reconfiguration flag to its neighboring nodes.

After the root node has completed its first broadcast, the remaining $\mathrm{N}-1$ nodes in the $\mathrm{N}$-node network broadcast one-by-one. However, during these N-1 broadcasts, the flag recipients will only perform the last two actions (Action3 and Action4). Each action is described in detail below.
Action1: Entering Recovery. Upon receiving the flag for the first time (initial broadcast from the root node), each node invalidates its routing table, freezes the pipeline progress of head flits, and sets its state as "recovering". The state will automatically switch back to "normal" in exactly $\mathrm{N}^{2}$ cycles, since the reconfiguration process is guaranteed to have completed by then. Once the node gets into recovery mode, each subsequent incoming flag will only invoke Actions 3, 4.

Action2: Tagging Link Directions. Once a node gets into recovery mode, up*/down* routing restrictions have to be applied. In up*/down*, links towards the root node (connecting to a node which is closer to the root) are up links, while links away from the root are down links. Links to a node of equal distance to the root (as the current node) can be either. During the initial broadcast by the root, a port that receives a flag connects to a node that is closer to the root (since the flag arrived there first), thus it is marked as up. Similarly, a port that forwards/sends a flag on is marked as down. The only conflict occurs when neighboring nodes with equal distance to the root node attempt to send the flag to each other in the same cycle. In this case, each node will receive the flag from a port while trying to send it to the same port. When this happens, up*/down* suggests that the direction of the corresponding link can be either up or down, so we set it based on the statically assigned nodeIDs: the node with the higher nodeID will mark the link as up; the other node will mark the link as down (shown in the Action2 diagram of Figure 2).

After this assignment, all the down $\rightarrow u p$ turns are disabled. This restriction inherits the deadlock freedom of up*/down*, as explained in Section VII-A. Though a number of paths are disabled, there is always at least one deadlock-free path that connects all nodes reachable from the root node. That is because the minimal route from any node to the root (up) and from there to any destination node (down) is always available.

Action3: Routing Table Update. During each broadcast, the broadcasting node communicates to other nodes how it can be reached. When nodes receive the broadcasted flag, they record the ports where the flag was received from in their own routing table, to learn how the broadcasting node can be reached. This requires the broadcasts to spread via enabled turns only, so that the recipient of the flag can follow the opposite path to reach the broadcasting node. The third Action of Figure 2 shows that the flag from node D's broadcast arrives to the current node via its North and East ports, thus these ports are marked in the routing table to lead to D.

Action4: Flag Forwarding. In the next cycle, the node broadcasts the flag only to those port(s) from which it did not receive a flag earlier and that correspond to enabled turns (a flag received from up link(s) is never broadcasted to up links, because this will enable a routing path with a down $\rightarrow$ up turn, as shown in the last diagram of Figure 2). Since forwarding a flag takes a single cycle, each broadcast will deterministically complete in at most $\mathrm{N}$ cycles ${ }^{4}$.

\footnotetext{
${ }^{4}$ Each broadcast is bounded to $\mathrm{N}$ cycles. The worst case scenario occurs when all nodes are connected in an open ring, and the longest broadcast from one end to the other requires $\mathrm{N}-1$ cycles.
} 


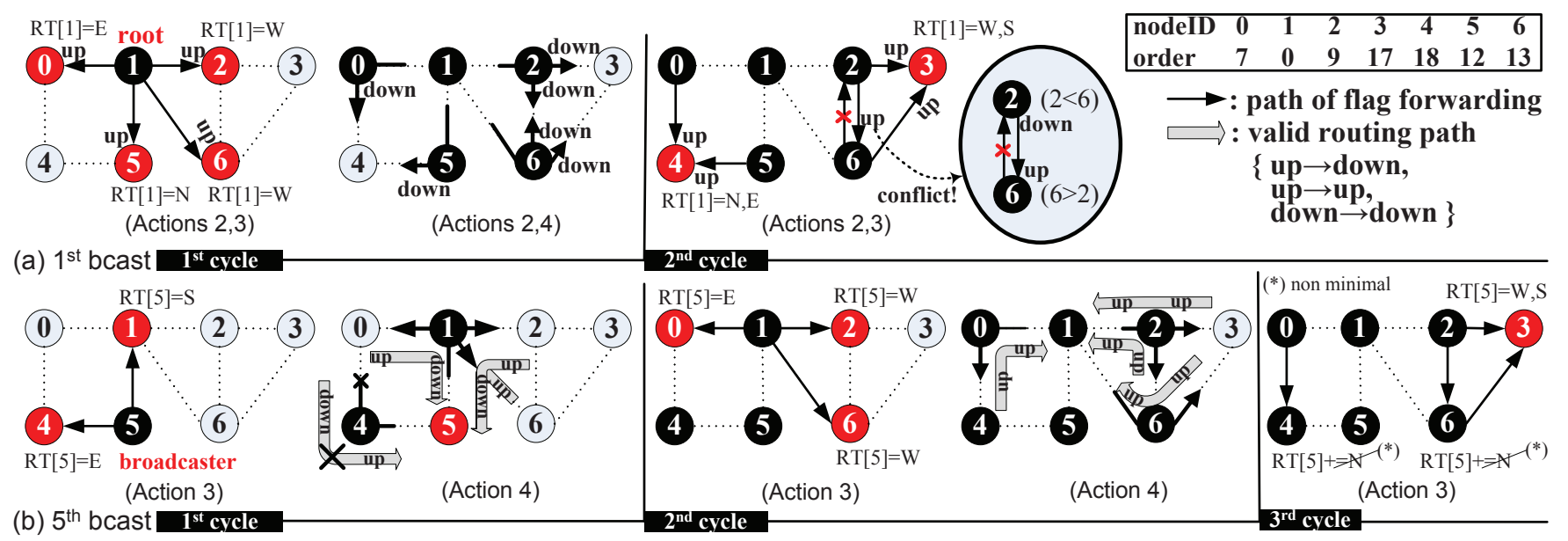

Fig. 3: Example of Ariadne reconfiguration. Initial broadcast by root node and 5th broadcast by Node5.

Completion of Reconfiguration. Reconfiguration is deterministically completed within $\mathrm{N}^{2}$ cycles since each node broadcasts once, and each broadcast takes at most $\mathrm{N}$ cycles. During this time, all routing tables are updated with resilient paths to any connected node, thus communication may be resumed and all nodes set their state back to "normal". After this point, any node can initiate a new broadcast upon detection of a link failure and invoke the reconfiguration process again. The head flits can now proceed in the pipeline, but since routes have changed (routing tables have been updated), they must restart from the route compute stage to find an alternative port that leads to the desired destination.

Walkthrough Example. An example of the reconfiguration process is shown in Figure 3. In this figure, we show a 7-node network connected in an irregular topology. Node1 detected a link failure, and initiates a broadcast. Figure 3a shows how each node receives the flag during the initial broadcast, marks the link(s) the flag was received from as up (Action2), fills the entry corresponding to the broadcasting node in its routing table (Action3), and then forwards it to its neighbors (Action4), while marking those link(s) as down (Action2). Node2 and Node6 receive the flag during the $1^{\text {st }}$ cycle and forward it to each other during the $2^{\text {nd }}$ cycle. To resolve this conflict, the node with the higher NodeID (i.e., Node6) marks the link as $u p$ and the other node (i.e., Node2) as down. We note that there is an implicit unique node ordering, shown in the table, which will be leveraged in our deadlock freedom discussion in Section VII-A.

Each subsequent broadcast can only follow paths that are consistent with the up*/down* restriction. As shown in Figure $3 \mathrm{~b}$, when Node5 is broadcasting, Node4 does not forward the flag to its North port ( $1^{\text {st }}$ cycle, Action4), because this would result in Node0 following an illegal path to reach Node5 (the Node $0 \rightarrow$ Node $4 \rightarrow$ Node5 turn is down $\rightarrow$ up). During Node5's broadcast, all nodes only perform Actions 3 and 4: they mark which port they should follow to route to Node5 in their own routing table, and then they forward the flag to all legal directions. Note that all nodes can reach Node5 via the root (up to the root Node1, then down to Node5). Also note that some nodes may use multiple output ports to reach Node5 (e.g., Node3 can either use its West or South port, since it received the flag from both ports during the $3^{\text {rd }}$ cycle), enabling adaptive routing. In this work, we only considered minimal paths for simplicity in adaptive routing. Thus, nodes that have already filled a routing table entry, ignore future flags for the same broadcasting node (for example, Node4 and Node6 do not record their North port as a potential path to Node5 during the $3^{\text {rd }}$ cycle, since this would lead to a nonminimal route). At runtime, the port with the highest number of available virtual channels among all valid (recorded) ports is selected to balance traffic density.

\section{B. Timing and Synchronization}

Section IV-A presented the reconfiguration algorithm. What has not been detailed so far is the timing of the reconfiguration: How does each node know when to broadcast so that there is no overlap between broadcasts? How does the recipient of a broadcast know the broadcasting node, since the only data broadcasted is a 1-bit flag? How do nodes know when the reconfiguration is completed? If two nodes concurrently detect a new fault, can they both become roots and initiate a broadcast? This section deals with these timing issues by introducing the notion of atomic broadcasts, where the cycle number ${ }^{5}$ indicates the ID of the broadcasting node.

Atomic Broadcasts. The idea of atomic broadcasts is to correlate the cycle number at which a broadcast is initiated to the broadcasting node's nodeID. Using the cycle number as a common reference point, all nodes are assigned different cycles for broadcasting, during which the remaining nodes are prevented from broadcasting for a window of $\mathrm{N}$ cycles (every broadcast is guaranteed to complete in $\mathrm{N}$ cycles, where $\mathrm{N}$ is the number of nodes). Each node will have to wait

\footnotetext{
${ }^{5}$ We assume a single synchronized global clock across the entire system. By the term "cycle", we refer to the count of the positive clock edges in a node (i.e., router and computation unit). It is not necessary that the communication system is controlled by the same clock as the computation units, but it has to be driven by a single clock.
} 


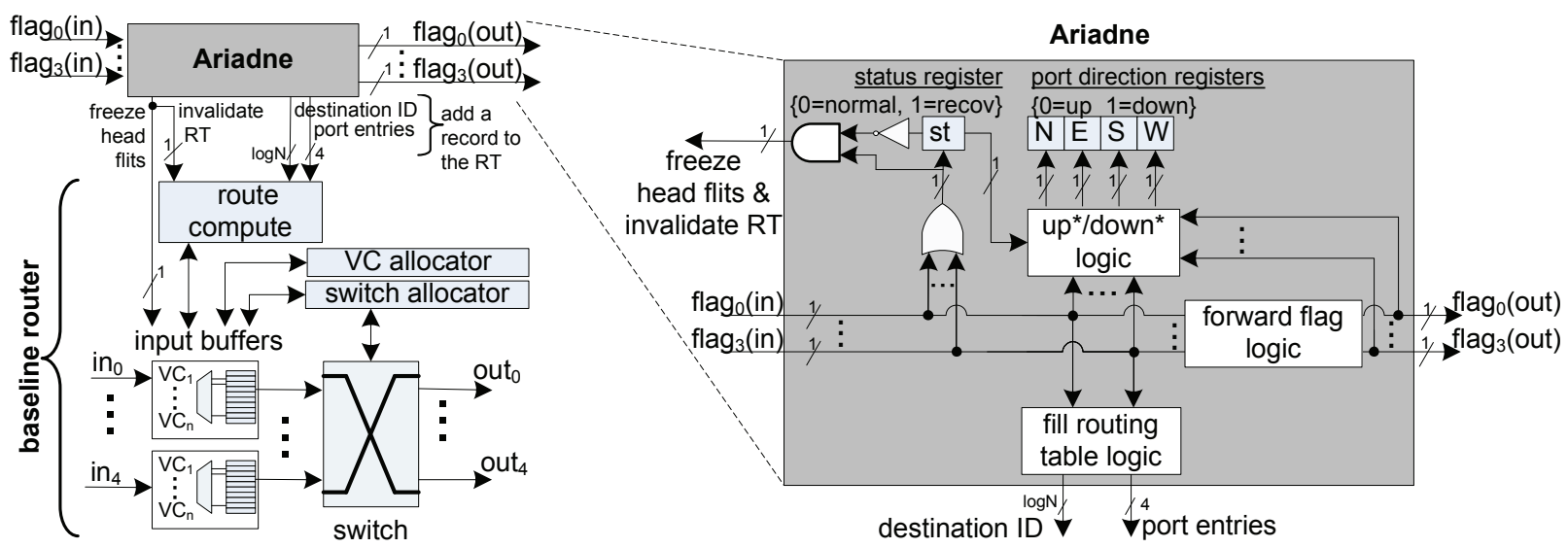

Fig. 4: Router Architecture. Ariadne's components shown shaded and expanded on the right part of the figure.

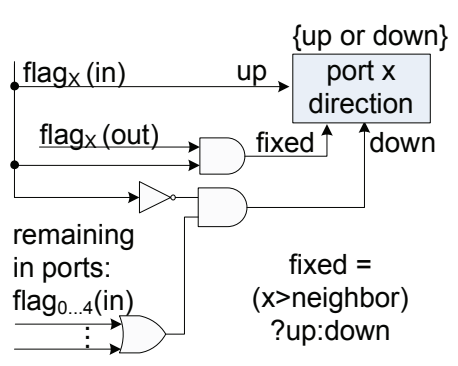

(a) up $^{*} /$ down* logic

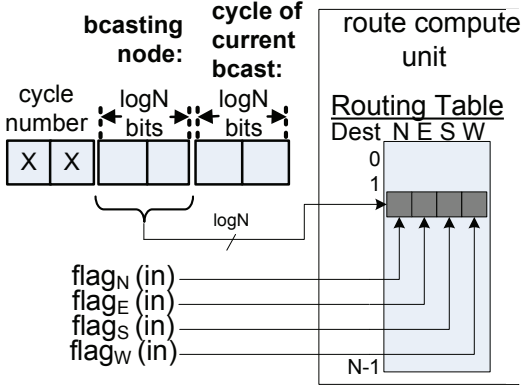

(b) fill routing table logic

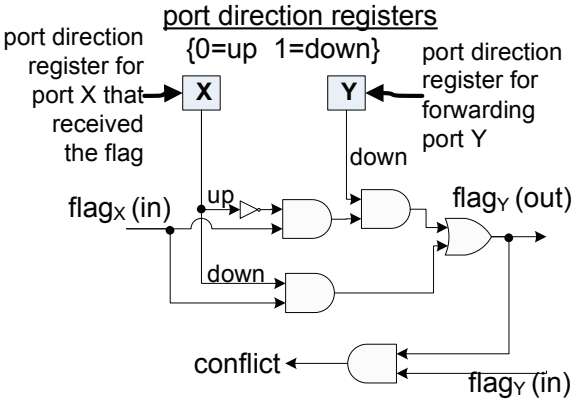

(c) forward flag logic

Fig. 5: Logic schematic for the building blocks of Ariadne's reconfiguration algorithm.

for its slot to broadcast, but during that slot no other node would be broadcasting, causing a collision. $\mathrm{N}$ slots need to be provided for all $\mathrm{N}$ nodes to broadcast, with slots looping around throughout execution. In other words, node $(\mathrm{X})$ 's Ncycle slot is always followed by node $((\mathrm{X}+1) \operatorname{modN})$ 's $\mathrm{N}$-cycle slot. The idea is similar to time-division multiplexing, where a number of signals physically take turns to transfer data on the same communication channel. Once the root node broadcasts, every node will deterministically broadcast during its first available slot; assuming that $\mathrm{R}$ is the root, nodes will broadcast in the order: $\mathrm{R}, \mathrm{R}+1, \ldots, \mathrm{N}-1,0,1, \ldots, \mathrm{R}-1$. Since $\mathrm{N}$ slots of $\mathrm{N}$-cycles are required for the reconfiguration to complete, reconfiguration requires precisely $\mathrm{N}^{2}$ cycles. Thus, all nodes can resume operation $\mathrm{N}^{2}$ cycles after receiving the initial broadcast, since by that time the process has been completed.

We note that if more than one node concurrently detects a fault, only the one who first receives a broadcast slot will become the root. The others will resign from becoming the root once they receive the root's broadcast. Our atomic synchronization ensures that no two nodes will ever simultaneously become the root. Once reconfiguration has been initiated, Ariadne will consider all detected faults in the network, independently of the node that managed to become root. Also, if a node is disconnected, it will not receive the root's broadcast, and will thus remain silent during its broadcast slot. Other nodes will thus not fill the corresponding entry in their routing tables (marking this node as unreachable).

\section{Router ARCHitecture}

To evaluate our solution, we considered a baseline router with a 5-stage pipeline ${ }^{6}$, as illustrated in the left part of Figure 4 , with the following units: a route-compute unit to determine the output port for a packet based on the final destination, a virtual channel allocator to reserve a virtual channel in the selected output port, a switch allocator to reserve the switch bandwidth, and the switch itself.

To implement Ariadne, we added a few gates, registers, and wires to the baseline router, as highlighted in the shaded portion of Figure 4. Assuming four bi-directional ports (four routing directions), Ariadne requires eight 1-bit wires (one for each port's direction) to transmit and receive the reconfiguration flag from all directions. Ariadne logic consists of a 1-bit status register to record the state ("recovering" or "normal"), and one 1-bit register per port to remember the port's direction (up or down). The logic to update the status register (Action1), to implement up*/down* (Action2), to fill the routing tables (Action3), and to forward the reconfiguration flag (Action4) are shown in the right part of Figure 4.

Figure 5 shows how these logic blocks are implemented. The up*/down* logic (5a) updates the port direction registers: receiving the flag from that port implies $u p$, receiving it from another port implies down, and sending and receiving at the

\footnotetext{
${ }^{6}$ Ariadne is orthogonal to the router pipeline. During normal operation, Ariadne is not active. During reconfiguration, the router pipeline is frozen, while Ariadne utilizes separate hardware to receive/broadcast flags.
} 
Network Architecture (GARNET)

\begin{tabular}{|l|l|}
\hline network topology & $8 \times 8$ 2D mesh \\
\hline memory controllers & 4 (chip corners) \\
\hline channel width & 64 bits \\
\hline router architecture & 5 -stage pipeline \\
\hline router ports, VCs & 5,2 (private) \\
\hline router buffers/port & 5 -flit/VC \\
\hline
\end{tabular}

\begin{tabular}{|l|l|}
\multicolumn{2}{|c|}{ System Configuration (GEMS) } \\
\hline processors & in-order SPARC cores \\
\hline coherence & MOESI protocol \\
\hline L1 caching & $\begin{array}{l}\text { private unified 32KB/node } \\
\text { ways:2 latency:3 cycles }\end{array}$ \\
\hline L2 caching & $\begin{array}{l}\text { shared distributed } 1 \mathrm{MB} / \text { node } \\
\text { ways:16 latency:15 cycles }\end{array}$ \\
\hline
\end{tabular}

Simulation Input
\begin{tabular}{|l|l|}
\hline synthetic traffic & $\begin{array}{l}\text { Uniform Random } \\
\text { TRranspose }\end{array}$ \\
\hline benchmark suite & PARSEC \\
\hline packet length & 5 \\
\hline simulation time & $100 \mathrm{~K}$ cycles \\
\hline simulation warmup & $10 \mathrm{~K}$ cycles \\
\hline
\end{tabular}

TABLE II: Simulated system configured in GEMS/GARNET.

same time implies that the direction should be determined by the routers' NodeIDs. The logic that compiles the routing tables $(5 b)$ sends to the routing unit the ID of the broadcasting node together with the input port(s) from which the flag was received, which indicate(s) the routing direction to the broadcasting node. The broadcasting node is extracted from $\log \mathrm{N}$ bits of the cycle counter, as will be detailed below. The routing tables consist of $\mathrm{N}$ Boolean entries (one for each destination) of four bits (indicating the four output ports). We note that during reconfiguration, all flags corresponding to minimal paths to a specific destination node will concurrently arrive at the same cycle; consequently, once a routing table record is set, it cannot be overwritten in later cycles (i.e., if the bit for at least one port is set to true in the routing table, all future records for that same entry are ignored). Finally, the logic responsible for forwarding the flag $(5 \mathrm{c})$ always forwards the flag if received from an input port marked as down; if the input port was marked as $u p$, then it only forwards if the corresponding output port is marked as down (Section IV-A).

Extracting the broadcaster ID from the cycle number. The hardware to implement this functionality is fairly simple. The $\log \mathrm{N}$ least significant bits of the cycle counter indicate the cycle number of the current broadcast slot (1 to $\mathrm{N}$ ). The next $\log \mathrm{N}$ most significant bits indicate which node is authorized to broadcast during the current slot (1 to N). We note that a cycle counter does not cost additional overhead, since it is already available in the performance counters of each processor core [1]. An example for $\mathrm{N}=4$ is shown in Figure 6, where the node that broadcasted the flag is indicated by bit $_{3}$ bit $_{2}$. A node can only initiate a broadcast at the first cycle $\left(\right.$ bit $\left._{1} \mathrm{bit}_{0}=00\right)$ of its turn ( $\mathrm{bit}_{3} \mathrm{bit}_{2}=$ NodeID) and it is guaranteed to complete this broadcast within 4 cycles (by bit $_{3}$ bit $_{2}=$ NodeID and bit $_{1}$ bit $_{0}=11$ ).

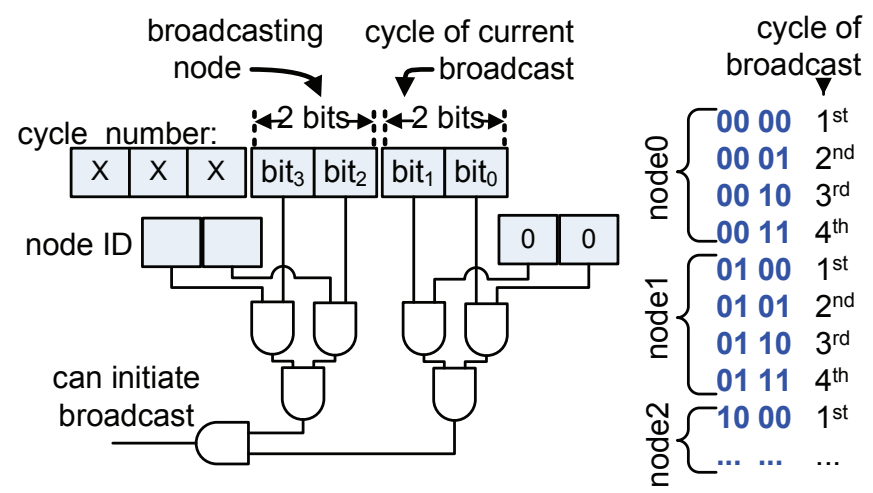

Fig. 6: Implementation of atomic broadcasts. Hardware that leverages the global clock to guarantee atomicity in broadcasts for an example 4-node $(\mathrm{N}=4)$ network.

\section{EXPERIMENTAL RESULtS}

In this section, we evaluate Ariadne to determine its effectiveness in faulty networks. We model faults with an accurate gate-level fault model described in Section II, where we map gate-level faults to link-level faults. Each simulation is performed 1,000 times, each time using a different injected fault configuration generated by our fault model (for 0 to 100 gate faults). We assume an ideal fault-detection mechanism, since fault-detection is orthogonal to reconfiguration. In our experimental setup, we evaluate the runtime performance (Subsection VI-A), reliability (Subsection VI-B), reconfiguration duration (Subsection VI-C), and hardware overhead (Subsection VI-D) of our solution. Ariadne is implemented in the Wisconsin Multifacet GEMS simulator [22] as part of the GARNET network model [2]. Additionally, two state-ofthe-art routing algorithms are implemented for comparison: the routing algorithm [14] of Vicis [15] and Immunet [25]. We configure GARNET to use an $8 \times 8$ mesh network (our simulation infrastructure is shown in Table II) and simulate synthetic traffic (Uniform Random and Transpose traffic), as well as the PARSEC benchmark suite [5].

\section{A. Performance Evaluation}

We consider two metrics to measure the performance of Ariadne on a faulty network: average latency and throughput. Latency is defined as the delay experienced by a packet from source to destination, while throughput demonstrates the rate of packets delivered (per cycle) in the entire network. First, we look at the zero-load latency for each of the three routing algorithms (Vicis, Immunet, and Ariadne), reflecting the steady-state latency of a lightly loaded network (0.01 flits injected per cycle per node, well below saturation). Each data point in Figure $7 \mathrm{a}$ is the average zero-load latency of 100 different topologies with the same number of faults.

We note that Ariadne's latency is consistently the lowest, at 43 cycles on average, compared to 58 cycles for Immunet and 97 cycles for Vicis. At 50 faults, the difference increases, with Ariadne showing a $43 \%$ latency improvement over Immunet and a $142 \%$ improvement over Vicis. Moreover, we note that the latency trend is strongly dependent on the algorithm, but not greatly affected by the traffic type, as shown by the closeness of the lines for each given algorithm. Vicis shows some interesting behavior here, increasing in latency as the number of faults increases. Upon further investigation, we found that this was caused by the occasional deadlocks encountered by the Vicis algorithm, which in turn trigger a 


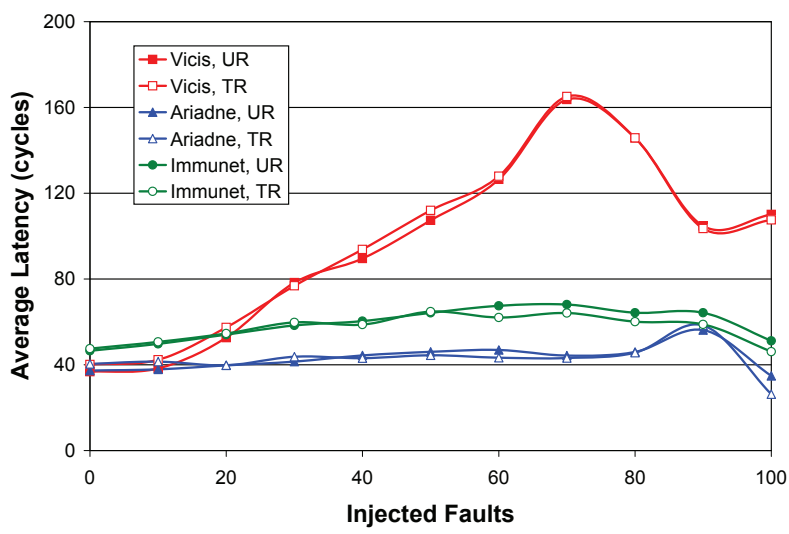

(a) Zero Load Latency. The average latency with a flit injection rate of 0.01 , plotted for varying number of faults injected.

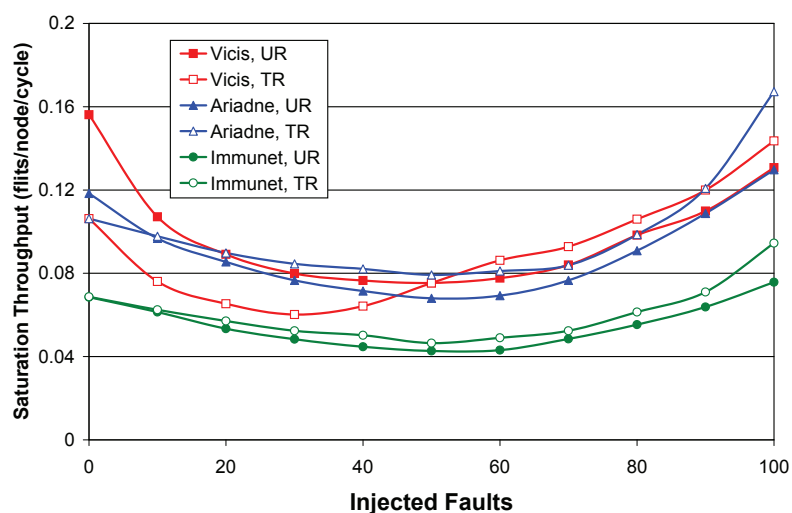

(b) Saturation Throughput (flits/node/cycle) reaches a minimum at 50 faults for all three algorithms. Then, it increases since packets route within small partitioned subnetworks.

Fig. 7: Performance on synthetic traffic

5,000-cycle timeout before dropping the deadlocked packets ${ }^{7}$. We note that Ariadne and Immunet never drop a packet. Ariadne maintains a reasonable constant latency, outperforming Immunet, since all of its virtual channels can route adaptively. In contrast, Immunet has one escape virtual channel restricted to route deterministically in a high-latency ring that goes through all surviving nodes two times (both directions) on average, independently of a packet's destination.

Figure $7 \mathrm{~b}$ plots the saturation throughput as a function of faults. We used numerical analysis to find the throughput value within a precision of 0.01 . For each number of faults, we performed this calculation for 100 different configurations. Notice that for fault rates up to about 50, saturation throughput decreases as the number of faults increases, as it can be expected, since the number of available paths decreases. This effect changes as the number of faults increases past 50, when throughput begins to increase. That happens because the network becomes partitioned, thus packets are routed a few hops within small subnetwork partitions, so overall throughput actually improves. For the same reason, the type of traffic does not critically affect the saturation throughput at high number of faults, since packets are restricted to route only within

\footnotetext{
${ }^{7}$ We did not implement a re-transmission mechanism for packets dropped by Vicis, but to keep the simulation going we instantly deliver the dropped packets to their destination.
}

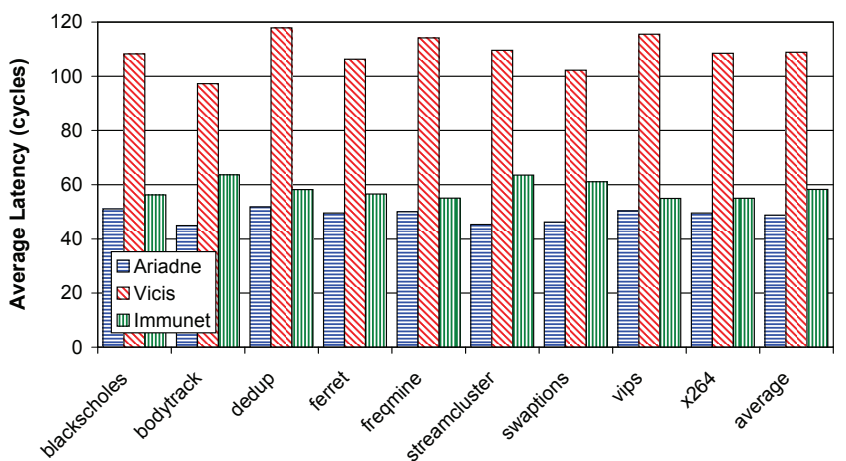

(a) Latency for $\mathbf{5 0}$ faults. Each bar shows the average latency for each benchmark and algorithm. The average latencies are shown in the rightmost bar.

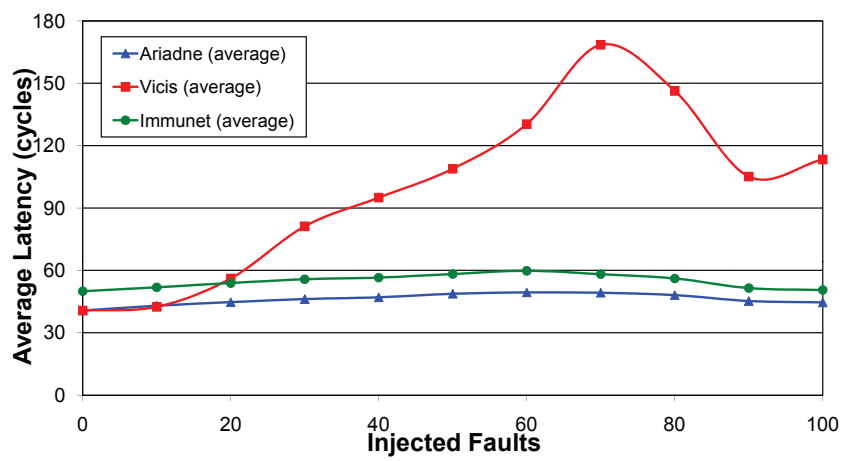

(b) Latency for variable number of faults. As the number of faults increases, Ariadne gives the best latency, while Vicis' latency quickly increases due to deadlocks.

Fig. 8: Performance for PARSEC benchmarks

these subnetworks. Note that the impact of traffic type on saturation throughput is not strong for Ariadne and Immunet. However, since Vicis is based on the turn model, which has naturally a higher saturation point, it more evenly spreads random traffic, particularly in few-faults situations, where the routing algorithm is closer to the turn model. We note that although based on the turn model, Vicis is deterministic, and uses a heuristic that chooses a minimal subset of available turns to reduce the probability of deadlock occurrence.

In addition to synthetic traffic, we also investigate the performance of our reconfigured network when running the suite of PARSEC [5] benchmarks. First, we ran each PARSEC benchmark on networks injected with 50 faults, the midpoint of our fault injection range. Measuring the average latency for packet delivery, we show the results for each algorithm in Figure 8a. Exhibiting trends similar to the random workload results, Ariadne outperforms Immunet and Vicis. We also observe that benchmarks that scale poorly beyond 16 cores (we simulated a 64-core system), such as bodytrack and streamcluster [5], entail more frequent messages between nearby nodes, resulting in lower average latency for Ariadne. This occurs because fewer threads, mapped to near-by cores, coordinate to complete the workload. In those cases, the latency difference between Ariadne and Immunet is highest $(45 \%)$, since Immunet leverages a fixed virtual ring that snakes through all nodes, so nodes that are physically close may have 


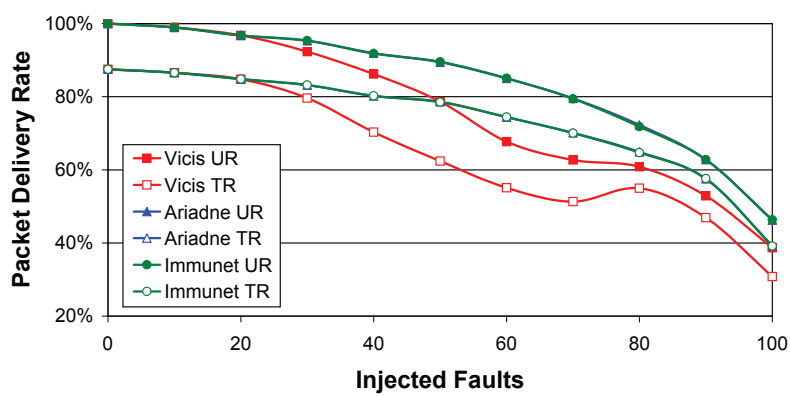

(a) Synthetic traffic.

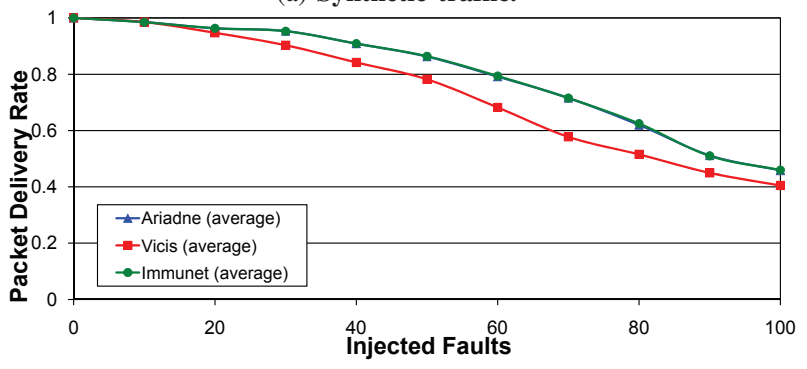

(b) PARSEC benchmarks.

Fig. 9: Packet delivery rate. The percent of packets delivered decreases as the number of faults increases. Undelivered packets in Ariadne and Immunet are due to not fully connected networks.

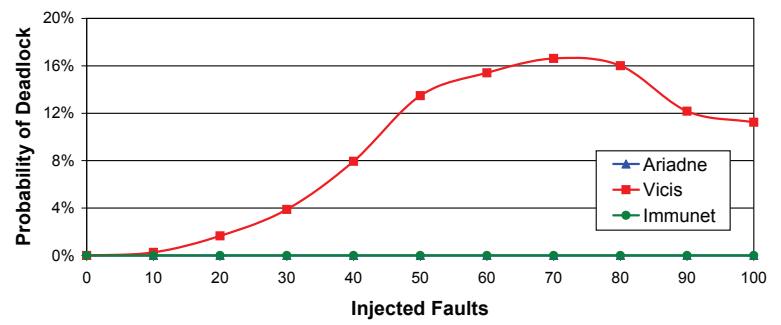

Fig. 10: Probability of deadlock over different fault configurations. Ariadne, Immunet are deadlock free (lines overlap at the $0 \%$ value), while Vicis is not.

to route through many hops via the ring. On the other hand, Vicis has its highest latency for benchmarks that scale well, such as dedup and vips, since long communication patterns are prone to creating cyclic routes and causing deadlocks.

In deepening our analysis while running PARSEC benchmarks, we also evaluate performance as the number of faults increases. Figure $8 \mathrm{~b}$ shows the average latency over all PARSEC benchmarks, as the number of injected faults ranges from 0 to 100 . Ariadne consistently provides the lowest average latency. The performance difference is most significant between Ariadne and Vicis under a large number of faults. We note that this figure is very similar to the zero load latency of synthetic traffic: that is because PARSEC benchmarks inject very low network traffic leading to low network stress.

\section{B. Reliability Evaluation}

Faulty networks are frequently not fully connected. The ability of an algorithm to maximize the connectivity of a faulty network is critical, since if there is no viable route between two locations, or if the routing algorithm has eliminated the only possible route to avoid deadlock, the packet can

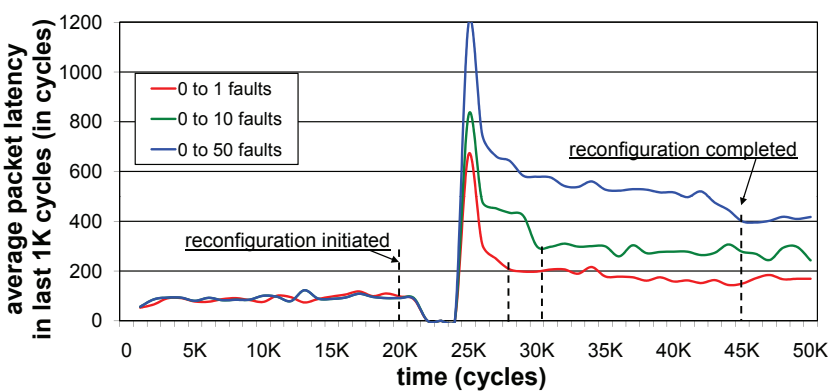

Fig. 11: Reconfiguration dynamics. Average packet latency over time, assuming uniform random traffic, 0.1 injection rate.

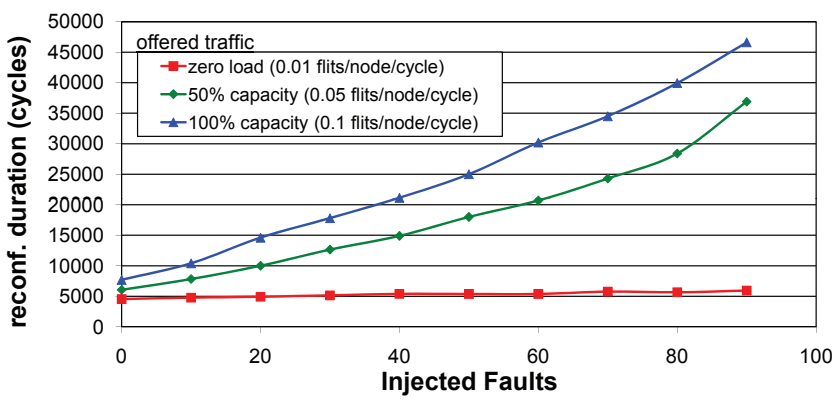

Fig. 12: Reconfiguration duration. Time required for packet latencies to stabilize after reconfiguration, for a variable number of faults/injection rates.

not be injected in the network. In Figures $9 \mathrm{a}$ and $9 \mathrm{~b}$ we show the packet delivery rate for both synthetic and PARSEC workloads. Packet delivery rate captures the number of packets delivered over the number of packets generated, and reflects the reliability of the network.

First, we observe that for all workloads, Ariadne and Immunet demonstrate identical delivery rates, delivering all packets injected in the network. Undelivered packets are solely due to the network being partitioned (see Section VII-E). Vicis, on the other hand, begins with performance similar to the other algorithms but, as faults increase, its delivery rate decreases. This occurs because packets are occasionally routed in circular routes, causing deadlocks and being dropped after a timeout. Figure 10 shows the probability of deadlock for an increasing number of faults (the lines for Immunet and Ariadne are constant on the $0 \%$ trend line).

\section{Reconfiguration Evaluation}

During reconfiguration, all packets in the network experience an additional delay of 4,096 cycles $\left(\mathrm{N}^{2}\right.$ cycles, for $\mathrm{N}=64$ ). That is because the route computation stage is stalled, so all head flits are frozen in the pipeline. Figure 11 shows the average latency (intervals of 1,000 cycles) over time for three reconfiguration procedures initiated in cycle 20,000: starting from a fault-free network, the curves correspond to the reconfiguration procedure for 1,20, 100 concurrent faults.

As shown in the figure, the average latency initially drops to 0 , since no packets are received for 4,096 cycles. After that, around cycle 25,000, the routing tables have been updated and all packets in the network can resume routing towards their destination. Since the Network Interface Controllers (NICs) 
were not stalled during reconfiguration, all injection queues will probably be full, resulting in increased contention right after normal operation is resumed. This explains the peaks in all three curves. The more the faults, the fewer the available paths to connect nodes, thus the higher the latency that these additional packets will experience (due to contention). Thus, we note that curves corresponding to topologies with more faults show longer times to drain packets generated during reconfiguration, and also stabilize at a higher average latency, since the new topology has lower capacity.

Figure 12 shows how the reconfiguration duration (time to drain packets generated during reconfiguration and stabilize to a new operating point) is affected by a variable number of faults and different injection rates. A low injection rate (zero load) will not generate a large number of packets during reconfiguration, so even with more faults (fewer available paths), there will be no congestion and the reconfiguration duration will only slightly increase (less than 1,000 cycles). For higher injection rates (comparable to the network capacity), the number of faults causes contention that lasts from 4,000 cycles (few faults) to 40,000 cycles (many faults, highly impaired network). Each point of the graph is an average over ten faulty (but fully connected) topologies.

Note that we do not anticipate link faults to be sufficiently frequent for reconfiguration duration to affect overall performance. The purpose of this graph is to provide quantitative insights on the reconfiguration process. Also, 50 concurrent transistor failures are more realistic in future 1000-core designs. The takeaway message of the figures is that Ariadne is viable under many concurrent faults, with reconfiguration duration scaling linearly.

\section{Hardware Overhead}

To determine the area impact of Ariadne in a router, we implemented its hardware structures in Verilog HDL. Beginning with a baseline 5-stage router design (Table II), we added the Ariadne logic illustrated in Figure 4. We also implemented the Vicis routing algorithm [14] and Immunet [25], whose major hardware additions are summarized in Table III. The Vicis routing algorithm requires the addition of a 64-entry static routing table, as well as modified routing logic to perform the routing heuristic. Immunet requires 3 additional routing tables (an adaptive, a deterministic, and a small safe table) and 4 wires per link. Each design was synthesized using Synopsys Design Compiler with an IBM $130 \mathrm{~nm}$ target library. The total area for the router equipped with Ariadne was measured at $2.761 \mathrm{~mm}^{2}$, which corresponds to an overhead of $1.97 \%$ (baseline router's area is $2.708 \mathrm{~mm}^{2}$ ); this overhead is slightly more than Vicis and three times less than Immunet.

\begin{tabular}{|l|r|l|c|r|}
\hline & wires & \multicolumn{1}{|c|}{ routing tables } & overhead & \%overhead \\
\hline \hline Ariadne & 1 & adaptive & $0.053 \mathrm{~mm}^{2}$ & $1.97 \%$ \\
\hline Vicis & 1 & deterministic & $0.040 \mathrm{~mm}^{2}$ & $1.48 \%$ \\
\hline Immunet & 4 & adapt., determ, small & $0.162 \mathrm{~mm}^{2}$ & $5.98 \%$ \\
\hline
\end{tabular}

TABLE III: Additional hardware summary.

\section{DISCUSSION}

In this section, we discuss how deadlock is avoided during normal operation (when using up*/down* routing, subsection VII-A) and during reconfiguration (Ariadne algorithm, subsection VII-B). Then, we elaborate on why existing offchip implementations of up*/down* would lead to excessive overhead if adapted on-chip (subsection VII-C). Finally, we explore whether Ariadne itself can fail (subsection VII-D) and what happens when nodes disconnect (subsection VII-E).

\section{A. Deadlock Avoidance (Normal Operation)}

Up*/down* has been proven in the literature to be deadlock free [27]. This is achieved by assigning a unique number (node order) to each node and disabling all increasing-order turns (down links), followed by decreasing order turns (up links). Note that the meaning of the terms "up" and "down" is reversed. Since the orders are unique, there can be no cycle without an increasing order followed by a decreasing order, thus the paths are deadlock free. Here, we prove that the way we assign link directions implies such a unique order. Assuming $\mathrm{N}$ nodes, each with a pre-assigned nodeID [0,N), and a root node with order 0 , we define the order of each NodeX that is connected to the root as:

\section{$\operatorname{order}(\mathbf{X})=\operatorname{distance}(\operatorname{root}, \mathbf{X}) * \mathbf{N}+\operatorname{nodeID}(\mathbf{X})$}

The distance is measured in hops, and corresponds to the minimal path that connects NodeX to the root. The table in Figure 3 shows an example of this order assignment, where Node1 is the root. This definition forces nodes that are closer to the root node to always have a lower order than nodes that are more distant (due to the multiplying factor $\mathrm{N}$ ). For nodes that are equally close to the root, their relative order is decided based on their statically assigned nodeID (since the first term of the equation above is the same): the node with the higher nodeID is considered to have higher order. These are the only two assumptions we used to assign a direction to each port, thus our direction assignment complies with the unique node ordering defined in the equation. Consequently, our nodes have a unique order and our routing paths are deadlock free.

\section{B. Deadlock Avoidance (Reconfiguration)}

The reconfiguration process replaces one routing function with another, by updating the routing tables in each node. As described in Section IV-A, all head flits freeze during this transition, thus the routing tables are not accessed and no packet may compute its route using the new routing algorithm until reconfiguration is completed. However, the body and tail flits may follow the route that the head flit has already reserved with the pre-fault routing paths. This section explores the dependencies between the old and the new routing function, which may create deadlocks once normal operation is resumed. The necessary condition for deadlock is a cyclic dependency of buffers, which is the result of routing in a cycle. During normal operation, cyclic routing is prevented by up*/down* as explained in VII-A, since a packet cached in an input buffer 
of an up link can never route to another up link (this would correspond to a down $\rightarrow$ up turn, see Action4 in Figure 2).

However, once the routing function is replaced, the directions of links may change. Thus, an input buffer of a down link, caching a packet that could be sent to any output (based on the old routing algorithm), may become an input buffer of an up link. If the only available route to destination is via an up link, this creates the condition for a deadlock. The deadlock occurs because of the post-fault routing algorithm inheriting a down $\rightarrow$ up dependency from a pre-fault routing path. We resolve such cases by ejecting the packet requesting the illegal turn to the NIC of the local router and re-injecting it to the network upon buffer availability. An additional dedicated flitbuffer per NIC is necessary to achieve this. This problem is present in all reconfiguration solutions: Immunet uses the same technique to prevent deadlock, while Vicis drops any packets requesting illegal turns. Using this technique, we save in-transit data and demonstrate a viable full-system reconfiguration solution incorporating Ariadne. In our simulations, we have verified that no packets are lost during reconfiguration and deadlock free operation is indeed preserved.

\section{Are Off-Chip Up*/Down* Schemes Applicable On-Chip?}

Section III mentions several off-chip resilient routing algorithms, most of which use up*/down* [27] as a baseline and modify the structure of its directed graph to balance traffic and enhance performance $[10,21,26]$. The simplest approach, the baseline up*/down* introduced by Autonet [27] in 1991, has been implemented in many high performance interconnects, such as Myrinet, InfiniBand, and Advanced Switching. However, these off-chip approaches cannot be adapted for on-chip implementation, due the high area overhead of its centralized reconfiguration algorithm.

In all prior implementations of up*/down*, the reconfiguration algorithm computes the routing tables in software with global knowledge of the surviving network. This software runs in a centralized management entity which is called mapper (in Myrinet), subnet manager (in InfiniBand), or fabric manager (in Advanced Switching). The underlying topology and spanning tree are communicated to this management entity, which performs the task of computing the new routing function in software. This procedure requires disabling a number of turns consistently with the spanning tree, and then iterating on the topology to find all the paths that each node can follow to route to every potential destination. Once a group of routing paths that guarantees deadlock-freedom and connectivity is found, the management entity sends a message to each node including its updated routing table.

We note that in earlier implementations of up*/down*, such as Autonet, the surviving topology is aggregated to a central node (root switch) and, from there, the topology and spanning tree are sent to every node to compute its routing table locally. This approach is still not distributed (we note that its authors name it "distributed"). We define as "distributed" a reconfiguration solution where: (1) all nodes are peers executing the same function, (2) there is only local knowledge of the surviving links. In contrast, in Autonet's reconfiguration (1) there is a single node (root-switch) assigned with the task of aggregating the surviving topology and delivering it to all nodes, while (2) each node requires global knowledge of all surviving links to compute its routing table.

Since these solutions require communicating the entire topology (in a reconfiguration packet) to a centralized location, each router has to maintain a reserved buffer and a reserved VC per input port to receive and forward this packet (the regular input buffers might be full holding in-transit packets during reconfiguration). This buffer should also incorporate additional logic to append to the reconfiguration packet the local router's functional links, their direction, and the router's ID. We note that any node might serve as the "management entity" (we cannot predict which nodes may become disconnected), thus every node should have reserved buffering for the entire reconfiguration packet, which accounts for 4 flits in a 64-node mesh, before interrupting the operating system to run up*/down*. We implemented this logic in Verilog HDL, synthesized it, and measured a $23.2 \%$ area overhead for our baseline router (Table II).

To avoid this overhead, we propose to reconfigure in a fullydistributed mode, where each node observes only a signal bit flag from its neighbors to fill its routing table, without any knowledge of the paths that the flags followed to arrive there, the topology, or spanning tree. Such a lightweight distributed implementation is not possible off-chip, since we require a global clock to provide specialized synchronization mechanisms for atomicity (detailed in Section IV-B).

\section{Can Ariadne Itself Fail?}

There is no resilient hardware that can guarantee $100 \%$ resilience. All resilient solutions we have described in this paper can fail if the resilient/recovery logic itself fails. Ariadne's Finite State Machine (FSM) uses a few gates to generate flags and update the routing tables. This FSM itself is susceptible to gate faults, while the 1-bit wires used to communicate the flags to neighbors can be corrupted by transient or permanent faults, resulting in the generation of a deadlock-prone routing algorithm. The most effective way to further protect small hardware structures, such as Ariadne, from failures is triple modular redundancy (TMR). In TMR, multiple identical structures perform the same operation and a voting system is used to feed the output with the result that the majority of the structures generated. That requires multiple copies of Ariadne's FSM in every router. Similarly, multiple identical flags (or ECC codes) must be transmitted to each neighboring router, which can utilize voting logic to recover the correct value of the flag in the case of faulty wires. We note that Ariadne's low overhead implementation makes TMR a very viable solution, since protecting all its hardware components with triple-redundancy would lead to an overall area overhead of less than $6 \%$. On the other hand, centralized software reconfiguration approaches would require the replication of the entire dedicated network for 
communicating the reconfiguration information and of the processor core executing it, which is not viable.

\section{E. What Happens When Nodes Become Disconnected?}

After a certain number of faults, there is a high probability that the network will become partitioned (split into disjoint clusters). Our reconfiguration solution will ensure that packet delivery is guaranteed within each partition, but cannot transfer packets among partitions. A network-only solution can identify this problem, but cannot recover the system. In such cases, the operating system will typically choose the largest partition of nodes and migrate all active threads there, marking all nodes that belong to other partitions as disconnected. To realize this without interruption of execution, the state and data of each active thread needs to be transferred from disconnected nodes to nodes of the active partition, while no available paths to connect these nodes exist in the underlying network. This is a complementary problem, recently tackled by DRAIN [13]. DRAIN is a recovery algorithm that uses dedicated emergency links to save the architectural state and cached data of disconnected nodes via cache-to-cache transfers.

\section{CONCLUSIONS}

We have presented Ariadne, an agnostic reconfiguration algorithm for NoCs, capable of circumventing large numbers of simultaneous faults, and able to handle unreliable future silicon technologies. Ariadne utilizes up*/down* for high performance and deadlock-free routing in irregular network topologies that result from large numbers of faults, and offers performance gains ranging from $40 \%$ to $140 \%$ (for 50 faults) during normal operation, compared to state-of-the-art fault tolerant solutions. It guarantees that if a path between two nodes exists, the reconfiguration algorithm will enable at least one deadlock-free path between them. Ariadne is implemented in a fully distributed mode, since nodes coordinate to explore the surviving topology, resulting in very simple hardware and low complexity. At $1.97 \%$ area overhead, Ariadne is a parsimonious solution for many-core processor designs of the future, enabling a trade-off between performance and reliable functionality on unreliable silicon.

\section{ACKNOWLEDGMENT}

The authors acknowledge the support of the Gigascale Systems Research Center and Interconnect Focus Center, research centers funded under the Focus Center Research Program (FCRP), a Semiconductor Research Corporation entity.

\section{REFERENCES}

[1] Intel core i7 processor. www.intel.com/products/processor/corei7.

[2] N. Agarwal, T. Krishna, L.-S. Peh, and N. K. Jha. Garnet: A detailed on-chip network model inside a full-system simulator. Proc. ISPASS, 2009.

[3] K. Aisopos, C.-H. O. Chen, and L.-S. Peh. Enabling system-level modeling of variation-induced faults in networks-on-chip. In Proc. DAC, 2011.

[4] S. Bell and et. al. Tile64 processor: A 64-core SoC with mesh interconnect. Proc. ISSCC, 2008.

[5] C. Bienia, S. Kumar, J. P. Singh, and K. Li. The PARSEC benchmark suite: Characterization and architectural implications. In Proc. PACT, October 2008 .
[6] P. Bogdan, T. Dumitras, and R. Marculescu. Stochastic communication: A new paradigm for fault-tolerant networks-on-chip. Hindawi VLSI Design, 2007.

[7] S. Y. Borkar. Microarchitecture and design challenges for gigascale integration. Proc. MICRO, 2004.

[8] S. Y. Borkar. Designing reliable systems from unreliable components: The challenges of transistor variability and degradation. IEEE Micro, 25(6):10-16, 2005.

[9] S. Chalasani and R. Boppana. Communication in multicomputers with non-convex faults. IEEE Trans. Computers, 1997.

[10] L. Cherkasova, V. Kotov, and T. Rokicki. Fibre channel fabrics: Evaluation and design. In International Conference on System Sciences, 1995.

[11] K. Constantinides, S. Plaza, J. Blome, B. Zhang, V. Bertacco, S. Mahlke, T. Austin, and M. Orshansky. Bulletproof: a defect-tolerant CMP switch architecture. Proc. HPCA, Feb 2006.

[12] W. J. Dally, L. R. Dennison, D. Harris, K. Kan, and T. Xanthopoulos. The reliable router: A reliable and high-performance communication substrate for parallel computers. In Proc. PCRCW, 1994.

[13] A. DeOrio, K. Aisopos, V. Bertacco, and L.-S. Peh. DRAIN: Distributed recovery architecture for inaccessible nodes in multi-core chips. In Proc. DAC, 2011

[14] D. Fick, A. DeOrio, V. Bertacco, D. Sylvester, and D. Blaauw. A highly resilient routing algorithm for fault-tolerant NoCs. Proc. DATE, 2009.

[15] D. Fick, A. DeOrio, J. Hu, V. Bertacco, D. Blaauw, and D. Sylvester. Vicis: a reliable network for unreliable silicon. In Proc. DAC, pages 812-817, 2009.

[16] C. J. Glass and L. M. Ni. Fault-tolerant wormhole routing in meshes without virtual channels. IEEE Trans. Parallel and Distributed Systems, 7(6), 1996.

[17] M. E. Gomez, J. Duato, J. Flich, P. Lopez, A. Robles, N. A. Nordbotten, O. Lysne, and T. Skeie. An efficient fault-tolerant routing methodology for meshes and tori. IEEE Computer Architecture Letters, 3(1), 2004.

[18] C.-T. Ho and L. Stockmeyer. A new approach to fault-tolerant wormhole routing for mesh-connected parallel computers. IEEE Trans. Computers, 53(4), 2004.

[19] J. Keane, S. Venkatraman, P. Butzen, and C. Kim. An array-based test circuit for fully automated gate dielectric breakdown characterization. In IEEE Custom Integrated Circuits Conference, pages 121-124, 2008.

[20] S.-P. Kim and T. Han. Fault-tolerant wormhole routing in mesh with overlapped solid fault regions. Parallel Computing, 23(13), 1997.

[21] M. Koibuchi, A. Funahashi, A. Jouraku, and H. Amano. L-turn routing: An adaptive routing in irregular networks. In International Conference on Parallel Processing, 2001.

[22] M. Martin, D. Sorin, B. Beckmann, M. Marty, M. Xu, A. Alameldeen, K. Moore, M. Hill, and D. Wood. Multifacet's general execution-driven multiprocessor simulator (GEMS) toolset. ACM SIGARCH Computer Architecture News, 33(4), 2005.

[23] A. Mejía, J. Flich, and J. Duato. Segment-based routing: An efficient fault-tolerant routing algorithm for meshes and tori. In Proc. IPDPS, 2006.

[24] M. Pirretti, G. Link, R. Brooks, N. Vijaykrishnan, M. Kandemir, and M. Irwin. Fault tolerant algorithms for network-on-chip interconnect. In Proceedings of the IEEE Computer Society Annual Symposium on VLSI, pages 46-51, 2004.

[25] V. Puente, J. A. Gregorio, F. Vallejo, and R. Beivide. Immunet: A cheap and robust fault-tolerant packet routing mechanism. ACM SIGARCH Computer Architecture News, 32(2), 2004.

[26] J. C. Sancho, A. Robles, and J. Duato. A flexible routing scheme for networks of workstations. In Proc. HiPC, 2000.

[27] M. Schroeder, A. Birrell, M. Burrows, H. Murray, R. Needham, T. Rodeheffer, E. Satterthwaite, and C. Thacker. Autonet: a high-speed, selfconfiguring local area network using point-to-point links. IEEE Journal on Selected Areas in Comm., 9(8), 1991.

[28] J. Srinivasan, S. Adve, P. Bose, and J. A. Rivers. The impact of technology scaling on lifetime reliability. Proc. DSN, pages 177-186, 2004.

[29] C.-C. Su and K. G. Shin. Adaptive fault-tolerant deadlock-free routing in meshes and hypercubes. IEEE Trans. Computers, 45(6), 1996.

[30] S. R. Vangal et al. An 80-tile sub-100-W teraflops processor in $65-\mathrm{nm}$ CMOS. IEEE Journal of Solid-State Circuits, 2008

[31] J. Wu. A fault-tolerant deadlock-free routing protocol in 2D meshes based on odd-even turn model. IEEE Trans. Computers, 52(9), 2003. 